Searching for binary coalescences with inspiral templates: detection and parameter estimation

This article has been downloaded from IOPscience. Please scroll down to see the full text article.

2009 Class. Quantum Grav. 26114009

(http://iopscience.iop.org/0264-9381/26/11/114009)

View the table of contents for this issue, or go to the journal homepage for more

Download details:

IP Address: 131.251.133.25

The article was downloaded on 04/04/2012 at 12:51

Please note that terms and conditions apply. 


\title{
Searching for binary coalescences with inspiral templates: detection and parameter estimation
}

\author{
Benjamin Farr ${ }^{1,2,3}$, Stephen Fairhurst ${ }^{1}$ and B S Sathyaprakash ${ }^{1}$ \\ ${ }^{1}$ School of Physics and Astronomy, Cardiff University, The Parade, Cardiff, UK \\ 2 Department of Physics, Rochester Institute of Technology, 85 Lomb Memorial Drive, \\ Rochester, NY 14623, USA \\ ${ }^{3}$ Center for Computational Relativity and Gravitation and School of Mathematical Sciences, \\ Rochester Institute of Technology, 85 Lomb Memorial Drive Rochester, NY 14623, USA
}

Received 15 January 2009, in final form 3 March 2009

Published 19 May 2009

Online at stacks.iop.org/CQG/26/114009

\begin{abstract}
There has been remarkable progress in numerical relativity recently. This has led to the generation of gravitational waveform signals covering what has been traditionally termed the three phases of the coalescence of a compact binarythe inspiral, merger and ringdown. In this paper, we examine the usefulness of inspiral only templates for both detection and parameter estimation of the full coalescence waveforms generated by numerical relativity simulations. To this end, we deploy as search templates waveforms based on the effective one-body waveforms terminated at the light-ring as well as standard post-Newtonian waveforms. We find that both of these are good for detection of signals. Parameter estimation is good at low masses, but degrades as the mass of the binary system increases.
\end{abstract}

PACS numbers: $04.30 . \mathrm{Tv}, 04.80 . \mathrm{Nn}, 07.05 . \mathrm{Kf}, 04.25 . \mathrm{dg}$

(Some figures in this article are in colour only in the electronic version)

\section{Introduction}

Several ground-based interferometric detectors are now in operation to detect gravitational waves. These include the Laser Interferometric Gravitational-wave Observatory (LIGO) at two sites in Livingston and Hanford, USA, and the Virgo detector in Cascina, Italy. They have recently completed a first science run at or close to design sensitivity and are sensitive to gravitational waves from coalescing binaries at distances of tens to hundreds of megaparsecs depending on the total mass and the mass ratio of the system. The broadband sensitivity (40$400 \mathrm{~Hz}$ ) of these detectors makes it possible to search for binaries with a rather large range of component masses from one to hundreds of solar masses. This range of masses includes both the neutron star binaries (which are known to exist) as well as neutron star-black hole and double black hole binaries (of which we have no observational evidence). 
In this paper, we test the efficiency of inspiral waveforms for the detection and parameter estimation of the full coalescence signal. We restrict our attention to two waveform families. The first of the families is the Fourier domain model, called TaylorF2 or SPA [1], which is an analytical approximation to the Fourier transform of the standard post-Newtonian (PN) [2-5] waveform (i.e., TaylorT3) computed using the stationary phase approximation. The waveform is computed to leading order in amplitude, whereby the higher harmonics of the signal are neglected. The highest PN order available in the LIGO Scientific Collaboration (LSC) code base [6] for this family is $(v / c)^{7}$ (i.e. $3.5 \mathrm{PN}$ order) in phase. A number of searches by the LSC for compact binaries of low masses (i.e. $M<25 M_{\odot}$ ) have used this model to second post-Newtonian order as optimal templates [7-10].

The second family we consider is the effective one-body (EOB) [11] model at four $\mathrm{PN}^{4}$ (i.e., $\left.(v / c)^{8}\right)$ order in phase but terminated at the light ring. As discussed below, the EOB model provides the full waveform, i.e., the inspiral, plunge, merger and ringdown waveform. In particular, the merger-ringdown waveform is obtained by stitching a superposition of quasinormal modes to the inspiral-plunge waveform at the EOB light ring [11-16]. In this study, however, we use the EOB model without the ringdown modes ${ }^{5}$. Thus, it captures some part of the coalescence signal, and is therefore better suited to search for higher mass signals. Here we will focus on the efficiency of our template bank to capture coalescence signals with the TaylorF2 and EOB models. In section 2, we discuss in greater detail the dynamics of binary black hole mergers and the PN and EOB models.

We test the efficiency and parameter estimation accuracy of the searches in two different ways. First, in section 3 we perform a Monte Carlo study of the efficiency of the TaylorF2 and EOB models to detect the full waveforms. Since the full waveform is not known over the entire parameter space, we make use of the EOB waveform calibrated to the numerical relativity results [14-18]. In this analysis, both the templates and simulated waveforms are calculated using only the leading-order amplitude term; thus higher harmonics are neglected. Then, in section 4, we perform a similar comparison making use of waveforms generated numerically. This study was performed on the Numerical INJection Analysis (NINJA) [19] data set which comprised simulated data for the LIGO and Virgo detectors with numerically obtained binary coalescence signals added. Further details of the numerical waveforms are available in [19], although it should be noted that the majority of these waveforms included several higher harmonics in addition to the fundamental mode. The original numerical results for the NINJA numerical waveform contributions are described in [13, 20-32] (where these are published results); the codes are described in [25, 28, 32-43].

\section{Binary black hole dynamics}

The evolution of a black hole binary is driven by back-reaction due to the emission of gravitational waves which causes the system to inspiral and merge. Confident detection of the emitted signal is greatly facilitated by an accurate understanding of the dynamics of the binary and the shape of the emitted waveform during inspiral and merger. The early evolution of a binary can be well modelled by the PN approximation during which the system slowly inspirals on an adiabatic sequence of quasi-circular orbits located at the (stable) minimum of the changing effective potential. In fact, for most of its lifetime a binary black hole can be accurately described by the balance of the rate of change of the binding energy with the energy

\footnotetext{
4 Post-Newtonian expansion is currently known only to $3.5 \mathrm{PN}$ order [2-5]; the unknown 4 PN coefficient is determined by matching the EOB to numerical relativity waveforms [11-16] (see below).

5 Throughout this paper, we will use EOB to refer to the EOB model truncated at light ring, and EOBNR to refer to the EOB waveform including the ringdown modes, calibrated to numerical relativity results.
} 
carried away to infinity by the radiation as given by the quadrupole formula. In fact, many of the ideas (effective potential, last stable orbit, etc) that are relevant when the component masses are greatly separated are still very useful in analytically modelling the system close to coalescence. However, they are perhaps not so useful or needed from the view point of numerical evolution.

As the system evolves, the effective potential changes and reaches a point when the potential transforms from one having a stable minimum and an unstable maximum to one having just an unstable minimum. After this, the system no longer possesses any bound orbits. The transition point, called the last stable orbit ${ }^{6}$ (LSO), occurs when the radius $r$ of the orbit (in Schwarzschild coordinates) approaches $r \sim 6 G M / c^{2}$, where $M$ is the total mass of the system. In terms of the dominant component of the emitted radiation, this corresponds to a gravitational-wave frequency of $f_{\text {insp }} \simeq 440 \mathrm{~Hz}\left(M / 10 M_{\odot}\right)^{-1}$. Therefore, for masses less than about $10 M_{\odot}$, only the inspiral stage of the coalescence lies in the detector's sensitive band of 40-400 Hz.

Once the system passes the LSO, the two black holes plunge towards each other and merge in about one orbital time scale of the LSO to form one single distorted black hole. This is the so-called merger phase which is amenable to analytic description by a clever re-summation of the PN approximation but more recently numerical relativity simulations have provided a better understanding of the merger phase and continue to provide new insights. The frequency of the waves during this phase changes rapidly from $f_{\text {merge }} \simeq 440 \mathrm{~Hz}\left(M / 10 M_{\odot}\right)^{-1}$ to $1200 \mathrm{~Hz}\left(M / 10 M_{\odot}\right)^{-1}$. During the late stages of the coalescence, the highly distorted black hole that results from the merger of the two parent black holes settles down to an axi-symmetric quiescent state by emitting its deformation in the form of gravitational waves. The radiation from this phase is well described by black hole perturbation theory and consists of a set of quasi-normal modes (often referred to as ringdown signal) whose fundamental frequency is $f_{\text {ring }} \sim 1800\left(M / 10 M_{\odot}\right)^{-1}$ when two equal mass non-spinning black holes merge to form a single black hole whose spin magnitude is estimated to be $J / M^{2} \simeq 0.7$. The first two overtones of this mode have frequencies of $\sim 1650 \mathrm{~Hz}$ and $\sim 1700 \mathrm{~Hz}$, for the same system.

\subsection{Search templates}

The foregoing discussion hints that binaries whose total mass is less than about $10 M_{\odot}$ can be detected by using templates that are described by the PN approximation. In fact, experience suggests that we could make do with the PN waveform as templates even when the total mass is as large as about $25 M_{\odot}$ and they have been used in the search for low-mass systems (i.e. systems with their total mass less than about $25 M_{\odot}$ ) in the data from LIGO and Virgo [10]. However, for higher mass black hole binaries (i.e. binaries with their total mass greater than about $25 M_{\odot}$ ) the merger of the binary occurs in the detector's sensitive band. At merger, the dynamics is no longer adiabatic and is, therefore, not well modelled by PN expansion. It has been a long standing aim of numerical relativity to generate the full waveforms for gravitational wave detection from higher mass black holes.

There has been significant progress recently in numerical relativity with several groups having successfully simulated the merger of two black holes (see, for example, [39, 42, 45] and the NINJA-related numerical relativity results cited earlier). The longest of these simulations

6 The LSO discussed in this paragraph refers to the test-particle limit of a binary when the mass ratio is very small; PN corrections and resummed models modify the location of the LSO [44]. However, these changes are unimportant to the present discussion where we are concerned with approximate numbers to determine the rough boundary between where a certain phase of the evolution is dominant. 
last for tens of orbits [30], and they cover different mass ratios and are beginning to explore the space of component masses with spins.

Nearly a decade ago, analytical work by Buonanno and Damour [11] extended the PN dynamics beyond the last stable orbit to calculate the merger dynamics. This analytical method, called EOB, computes the dynamics up to the light ring of the effective potential and the waveform can be computed for separations larger than about $r \simeq 2.2 \mathrm{M}$. In this work we have used the EOB waveform terminated at the light ring as search templates. The EOB formalism, however, provides the full waveform, including the merger-ringdown portion which is attached at the EOB light ring. Moreover, the availability of numerical relativity simulations has helped in fixing certain unknown higher-order (4 PN) terms in the EOB model by fitting the analytical waveform to numerical relativity. The current implementation of the full EOB model in LAL uses the 4 PN order parameter obtained in [17] by calibrating the EOB model to the Goddard-NASA numerical simulations, and three ringdown modes (i.e., the fundamental mode and two overtones). We note that, more recently, the EOB model has been further improved by calibrating it to more and more accurate numerical simulations $[14-16,18]$ which will form the basis of future searches. In the current study, EOBNR is used to calibrate the efficiency of the inspiral models. While the higher harmonics of both the EOB and EOBNR models have been calculated, they have not yet been implemented in the code; therefore, we use restricted waveforms for both EOB and EOBNR throughout the paper.

Ajith et al [46, 47] have used a phenomenological approach to match the inspiral phase from the PN approximation to the merger and ringdown from numerical relativity. Here, a hybrid waveform obtained by stitching together the PN inspiral waveform to the numericalrelativity inspiral, merger and ringdown waveform is first built. Then, the phenomenological waveform in the frequency domain is constructed by requiring closeness to the hybrid waveform. In the long run, it is likely that these full waveforms will be used as templates to search for inspiral signals in gravitational wave detectors.

In order to test the efficiency of EOB and TaylorF2 families, we will use the EOBNR waveform as our 'true' waveform and see how well these partial waveforms perform in both detection and parameter estimation.

\subsection{An example waveform}

Figure 1 shows the waveform expected from a pair of non-spinning $10 M_{\odot}$ black holes during the last $50 \mathrm{~ms}$ before merger. The left panel shows the time-domain waveform $h(t)$ and the right panel compares the time-domain waveform with the signal 'perceived' by the initial LIGO detector whose noise has been whitened. In other words, the right panel plots whitened template $q(t)$ given by

$$
q(t)=\int_{-\infty}^{\infty} \frac{H(f)}{\sqrt{S_{h}(|f|)}} \exp (-2 \pi \mathrm{i} f t) \mathrm{d} f,
$$

where $H(f)=\int_{-\infty}^{\infty} h(t) \exp (2 \pi \mathrm{i} f t) \mathrm{d} t$ is the Fourier transform of the time-domain signal and $S_{h}(f)$ is the one-sided noise power spectral density of initial LIGO. In the left panel, the inspiral part of the signal given by EOB dynamics is shown in the black solid line followed by the plunge, merger and ringdown phases in the red dashed line. Note that although the time-domain signal is dominated by the plunge, merger and the ringdown phases, the detector noise spectral density (i.e., $S_{h}(f)$ ) suppresses them, making the inspiral phase more dominant. For systems with greater masses, more of the merger phase appears in band. For systems with total mass larger than about $80 M_{\odot}$ the merger and ringdown signals begin to dominate over the inspiral phase. For such systems it is important to deploy EOBNR templates. As a result, we cannot expect our template families to do well in capturing high-mass binaries. 

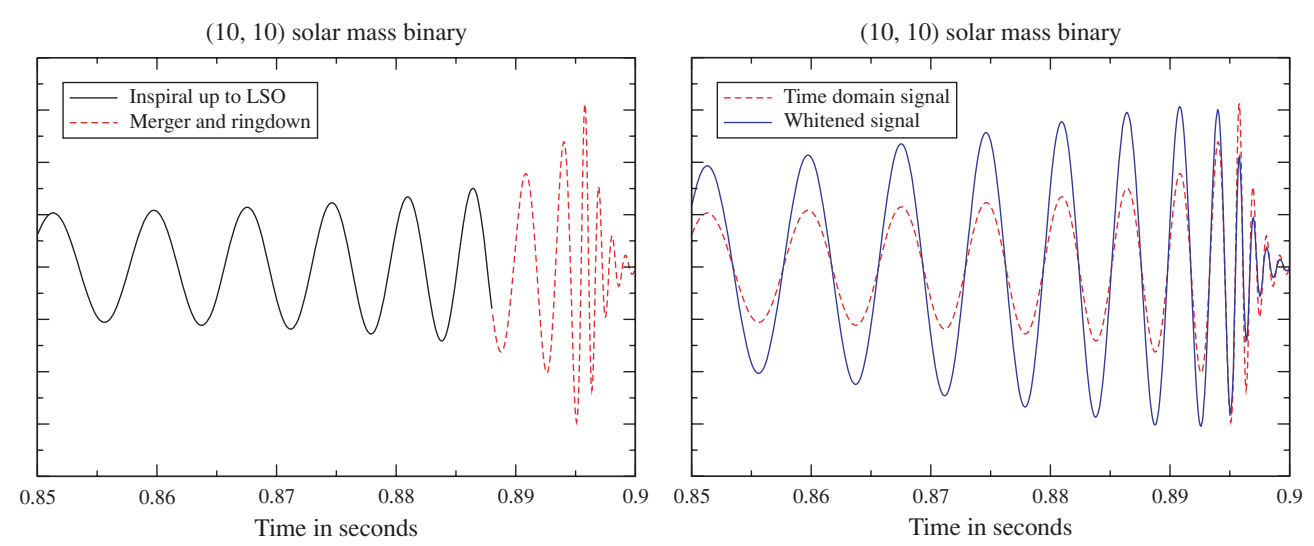

Figure 1. An example of the EOBNR waveform for a binary consisting of two equal mass, non-spinning black holes each $10 M_{\odot}$. The EOB dynamics allows the computation of the inspiral (left panel, black solid line) and plunge (left panel, roughly the first two cycles of the red dashed line) phases but the merger and ringdown waveform (left panel, latter part of the red dashed line) is stitched to the end of the plunge phase by matching the amplitude of the waveform and its first two derivatives by using a superposition of the fundamental quasi-normal mode and its first two overtones. The right panel compares the time-domain signal (red dashed line) with the signal whitened using the initial LIGO power spectrum (blue solid line).

\section{Bank efficiency}

Matched filtering, the data analysis technique used in most searches for binary black holes, is pretty sensitive to the phasing of the signal, which in turn depends on (a subset of) the source parameters. In the case of non-spinning black holes on a quasi-circular orbit, the only parameters that we must consider are the two masses. When restricting to the dominant harmonic of the signal, the location of the binary on the sky, the distance to the source, the polarization of the wave, etc, are not important for a single detector as they simply affect the amplitude of the waveform. Although we will not know the time at which the binary merges nor the phase of the signal at that epoch; these parameters need not be explicitly searched for [1], and are easily extracted in the process of maximizing the cross-correlation of the template with the data.

\subsection{Template bank}

Our goal in this section is to study the efficiency of the two template families in detecting binary black hole coalescences. To this end, we first set up a template bank-a set of points in the parameter space of the component masses. A geometric algorithm described by Babak et al [48] is used to generate the template bank, and it is the same algorithm irrespective of which family of waveforms is used to filter the signals. The bank is designed to cover the desired range of component masses of the binary. In addition to the range of the component masses, our template bank algorithm requires us to specify a parameter called the minimal match, MM. The minimal match is the smallest overlap guaranteed between a signal with random source parameters and the template nearest (in the geometrical sense) to it in the parameter space. The template placement algorithm chooses a hexagonal grid in the twodimensional parameter space of the component masses, and it is an optimal algorithm in the sense that it gives the smallest number of templates possible for a given minimal match [49]. 
Assumptions made in the construction of the template placement algorithm imply that this will be strictly true only when (a) the templates and signals belong to the same family, and (b) the ending frequency (i.e., the LSO or light ring depending on the waveform in question) is greater than the upper end of the sensitivity band. The latter condition further implies that we can hope to achieve overlaps of MM or greater only for waveforms whose total mass is smaller than a certain value depending on the detector bandwidth; in the case of initial LIGO this is $10 M_{\odot}$. We have chosen $\mathrm{MM}=0.95$. However, since our templates and signals belong to different PN approximations we cannot expect to achieve this overlap even for total mass less than $10 M_{\odot}$.

Having constructed a template bank for either the TaylorF2 or EOB waveforms, we then test it using signals from the EOBNR family. In particular, we generate an EOBNR signal with the values of its masses, the epoch and the phase at coalescence, all chosen randomly, but in a given range. Since the EOBNR extends beyond the LSO, it is possible to generate signals with the total mass in the range $[10,300] M_{\odot}$ and minimum component mass of $5 M_{\odot}$. We performed simulations for both the TaylorF2 and EOB templates over a restricted range of the parameter space and an additional EOB analysis over a much larger range. Specifically, in the case of TaylorF2 we set up a template bank with the total mass in the range $[6,80] M_{\odot}$, with minimum component mass of $3 M_{\odot}$, and tested it with signals with total mass in the range $[10,72] M_{\odot}$. Although the TaylorF2 model is not expected to be a good approximation above about $\sim 25 M_{\odot}$, we are using it to much higher mass. For the EOB family truncated at light ring, we performed two separate analyses. The first had templates covering the range $[6,120] M_{\odot}$, minimum component mass $3 M_{\odot}$ and injected signals with total mass in the range $[20,80] M_{\odot}$. Since the EOB waveforms extend to a significantly higher frequency than the standard post Newtonian ones, we also performed an analysis with templates of total mass in the range $[60,400] M_{\odot}$ and minimum component mass of $30 M_{\odot}$. In this case the template bank consisted of only nine templates. The simulated signals had total mass between 70 and $300 M_{\odot}$.

Next, for each point in the template bank we generate waveforms from our template families (EOB and TaylorF2) and measure their overlap with the random signal. The overlap $\mathcal{O}_{k}$ of the $k$ th template $q_{k}\left(t ; m_{1}^{k}, m_{2}^{k}\right)$ and the signal $h(t)$ is defined by

$$
\mathcal{O}_{k}\left(m_{1}^{k}, m_{2}^{k}\right)=\max _{t} 2 \int_{f_{l}}^{f_{u}}\left[H(f) Q_{k}^{*}\left(f ; m_{1}^{k}, m_{2}^{k}\right)+H^{*}(f) Q_{k}\left(f ; m_{1}^{k}, m_{2}^{k}\right)\right] \mathrm{e}^{-2 \pi \mathrm{i} f t} \frac{\mathrm{d} f}{S_{h}(f)},
$$

where $H(f)$ and $Q(f)$ are the Fourier transforms of $h(t)$ and $q(t)$, respectively, and $Q^{*}$ is the complex conjugate of $Q$. This allows us to compute the maximum overlap between our template waveforms and a random signal ${ }^{7}$. This process is repeated for 1000 different realizations of the random mass parameters and the maximum of the overlap over the entire template bank is recorded in each case. We will now discuss the results of these simulations.

\subsection{Efficiency for detection}

Figure 2 plots the results of our simulation. The left panel shows the (maximum) overlaps of the TaylorF2 template bank with random signals, one dot for each trial. The right panel shows the same but for the EOB template bank. The TaylorF2 model has overlaps larger than $90 \%$ for only signals whose total mass is less than about $22 M_{\odot}$. The overlap falls off quickly for masses larger than this, reaching slightly more than 0.5 when the total mass is about $50 M_{\odot}$.

7 For the sake of saving space we have not discussed the maximization over the phase of the signal. This can be found, for instance, in [1]. 

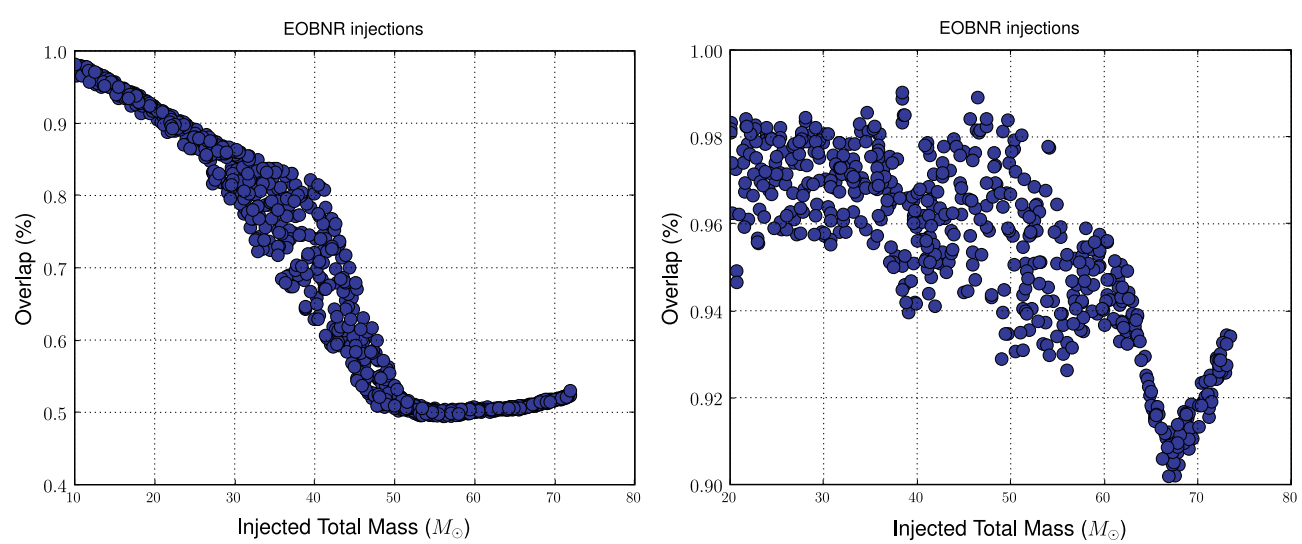

Figure 2. These plots depict the efficiency of the TaylorF2 (left panel) and EOB (right panel) template banks in detecting the coalescence waveforms assumed to be well represented by EOBNR. Each dot corresponds to the overlap of a random EOBNR signal maximized over the template bank consisting of TaylorF2 or EOB waveforms.

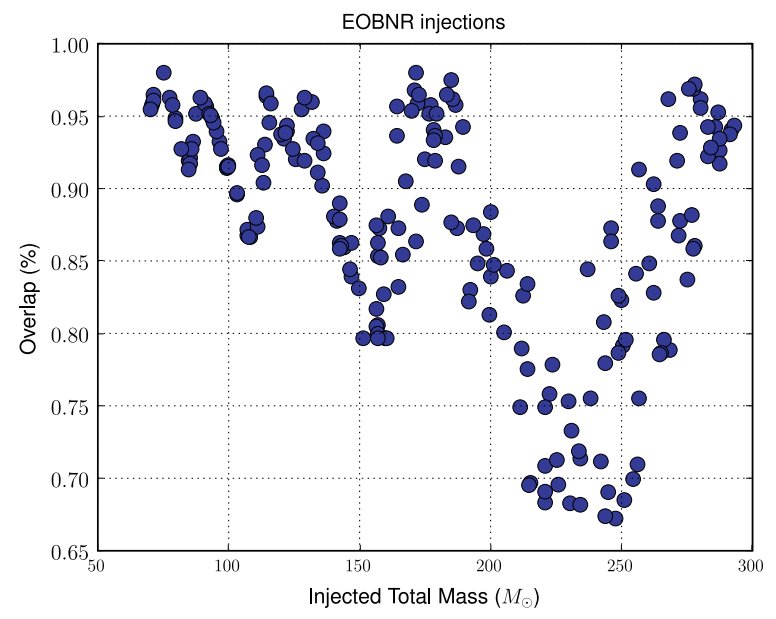

Figure 3. This plots depict the efficiency of the EOB template bank generated for the mass range $[60,400] M_{\odot}$ in detecting the coalescence waveforms assumed to be well represented by EOBNR. Each dot corresponds to the overlap of a random EOBNR signal maximized over the template bank consisting EOB waveforms.

The fact that the overlap remains unchanged beyond $50 M_{\odot}$ is probably due to the TaylorF2 template matching the later merger or ringdowm part of the EOBNR waveform. In contrast, the EOB model achieves an overlap greater than $90 \%$ over the entire mass range. This is due to the fact that the EOB waveform extends to the light ring.

Figure 3 shows the overlap obtained using the high-mass EOB template bank and an extended range of EOBNR simulated waveforms. We obtain overlaps of better than $85 \%$ for systems whose total mass is less than $150 M_{\odot}$, and the overlaps remain more than $65 \%$ even for systems with total mass less than $200 M_{\odot}$. As discussed at the beginning of section 3.1, this can be due to two effects: the difference between the signal and template waveforms, and the fact that the bank is constructed assuming the waveform's ending frequency is above 
the detector's sensitive band. For high masses, the waveform will terminate in the detector's sensitive band, and therefore the template bank will not achieve the desired minimal match. The oscillatory behaviour seen in this case is due to the small number of templates. In this range of total mass, there are only a handful of templates in the entire bank; as a result, a single template might be available for a large range of masses, causing the overlaps to swing up and down as the total mass is increased.

The overlaps are surprisingly high, especially considering that there are only nine templates in the bank. It is possible that the very high mass EOB signals are very short in the detector band (perhaps a cycle or two) and the abrupt cutoff of the template as a result of termination at the light ring could bleed power into a frequency region where there is no power in reality ${ }^{8}$. This spurious template power may lead to large overlaps with the merger and ringdown parts of the EOBNR waveforms. TaylorF2 does not suffer from this predicament. This is because TaylorF2 is generated in the Fourier domain and the abrupt cutoff of the signal does not cause any problem in the frequency domain, and we are unconcerned with spurious effects in the time domain as they occur outside the region of our interest.

\subsection{Efficiency for parameter estimation}

The matched filtering statistic gives the likelihood for a signal to be present in the data as opposed to the data being pure background noise. The parameters of the template which maximize the likelihood are maximum likelihood estimates. Having determined the efficiency of our template banks in capturing the coalescence signals, we next consider how good they are in measuring the signal parameters in the maximum likelihood sense. If the template waveforms and the signal they are intended to detect both belong to the same family then in the limit of large signal-to-noise ratio the distribution of the maximum likelihood estimates will be centred on the true signal parameters. We are in a situation wherein the template waveforms and the signals they are intended to recover belong to different approximations. Therefore, one can expect a systematic bias in the estimation of parameters.

To gauge the reliability of the two families in estimating the parameters of the true signal we make use of the results of the simulation from the previous section. This simulation computed the overlap of the templates with the signals in the absence of any noise. Therefore, the parameters of templates that maximized the overlap when compared to the true parameters of the EOBNR signal give a measure of the systematic bias in parameter estimation due to the difference in the waveform families representing the templates and the signal. Figure 4 shows the measured total mass of the template ( $y$-axis) versus the true total mass of the EOBNR signal ( $x$-axis) and the colour is determined by the corresponding overlap.

Both the TaylorF2 and EOB models provide good parameter recovery as well as good overlap for simulated signals with a total mass less than $30 M_{\odot}$. At masses higher than this, the TaylorF2 waveforms achieve a significantly lower overlap, although parameter estimation remains good up to a total mass of about $50 M_{\odot}$. Above this mass, all simulated signals are recovered by a template of mass $35 M_{\odot}$. Interestingly, this template will terminate around $120 \mathrm{~Hz}$ which is close to the most sensitive frequency of the LIGO detector. Thus, it seems likely that the inspiral template is picking up power from the merger and ringdown parts of the EOBNR waveform. For the EOB templates, the overlap does drop off somewhat for higher masses, but the parameter recovery remains reasonable throughout, with a slight tendency to underestimate the total mass of the signal. For higher masses, figure 5 shows the parameter

\footnotetext{
8 The spurious power is in itself not a bad thing but large noise glitches in the region where there is spurious power could cause false alarms. This is especially the case when the detector noise is contaminated by large amplitude non-stationary noise glitches.
} 

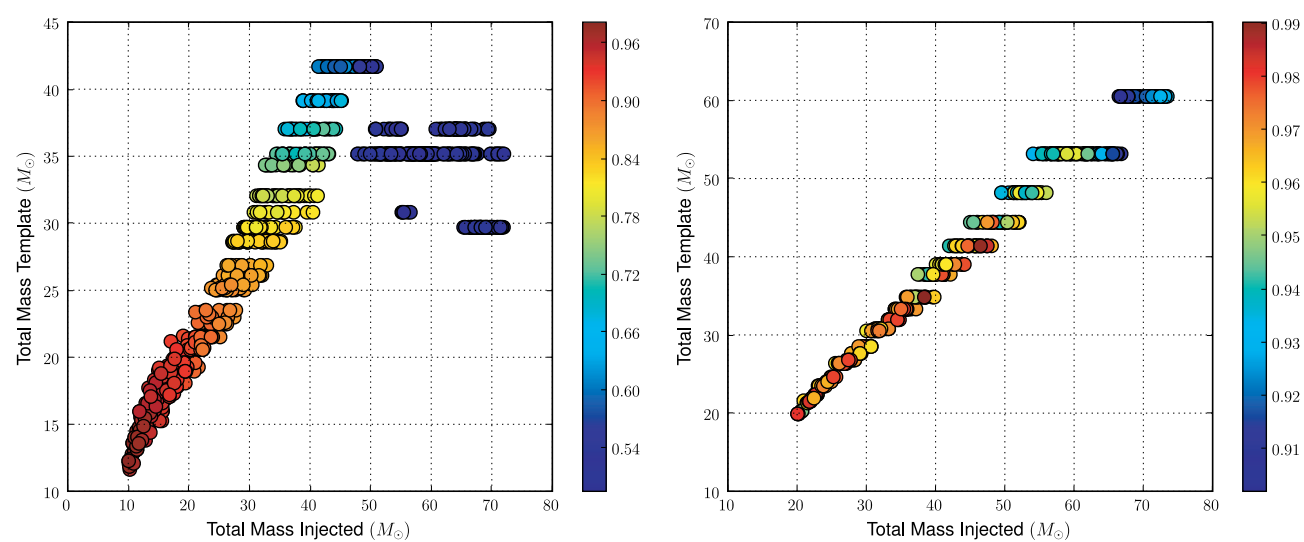

Figure 4. Parameter estimation accuracies for the TaylorF2 (left panel) and the EOB (right panel) models. The total mass of the injected EOBNR signal is plotted versus the total mass of the template that obtained the best overlap. The colour of the plotted point is determined by the overlap between signal and template. Clearly, there is a positive correlation between the injected and measured masses in the case of TaylorF2 model, with the spread in the measured values becoming larger at higher masses. Additionally, for higher masses the overlap decreases. Interestingly, all simulated signals with the total mass greater than $50 M_{\odot}$ are recovered with the same template of mass $35 M_{\odot}$. The EOB templates show a similar correlation between simulated and recovered masses. However, since the EOB templates extend to higher frequency, the EOB templates are capable of accurately recovering the higher mass simulations with accurate mass parameters and an overlap greater than $90 \%$.

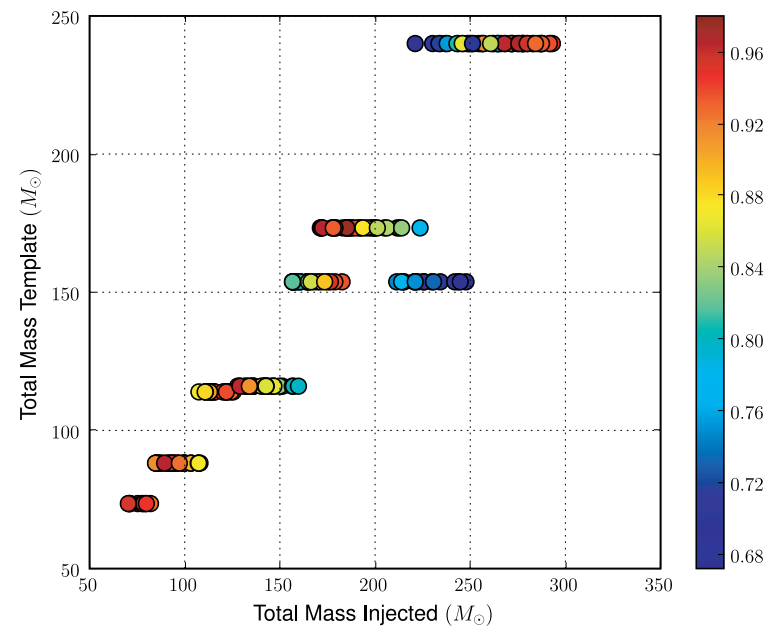

Figure 5. Parameter estimation accuracies for high masses using the EOB templates. The total mass of the injected EOBNR signal is plotted versus the total mass of the template that obtained the best overlap. The colour of the plotted point is determined by the overlap between signal and template. Even for high masses, there is a good correlation between the simulated and recovered masses. The discreteness of the EOB template bank is clearly seen from the limited set of recovered masses, and indeed this seems to account for the majority of poor overlaps.

estimation accuracy for the EOB waveforms. Despite the fact that there are only a handful of templates in the bank, the parameter recovery is reasonable. 
Table 1. Results of inspiral search for NINJA waveforms. There were 126 injections performed into the data. The table above shows the number of injections which were recovered using the two waveform families. The EOB search shows a significantly higher sensitivity than the TaylorF2 waveforms evolved to LSO. Note that virtually all simulations which pass the initial coincidence requirement also survived the signal consistency checks.

\begin{tabular}{lll}
\hline Template & TaylorF2 & EOB \\
\hline Freq. cutoff & LSO & Light ring \\
PN order & $2 \mathrm{pN}$ & $2 \mathrm{pN}$ \\
Found inj, single detector $(\mathrm{H} 1, \mathrm{H} 2, \mathrm{~L} 1)$ & $72,43,66$ & $91,64,82$ \\
Found inj, coincidence & 59 & 83 \\
Found inj, coincidence + signal vetoes & 59 & 80 \\
\hline
\end{tabular}

\section{NINJA results}

The Numerical INJection Analysis project was a mock data challenge, where the data were generated at the design sensitivity of the initial LIGO and Virgo detectors, and numerical relativity waveforms provided by a number of groups were added to the data. A number of data analysis methods were applied to the data, and the results of the NINJA project are available elsewhere [19]. For the NINJA analysis, we performed several runs through the data using the LSC's compact binary coalescence (CBC) analysis pipeline. Here, we restrict our attention to two runs through the data which are similar to the TaylorF2 and EOB analyses described in the previous section. This allows us to investigate the issues of detection and parameter estimation using these templates to search for full waveforms obtained from numerical relativity. The results are similar to those obtained in the previous section, namely that the EOB search has a greater efficiency than the TaylorF2 search, but that both can detect high-mass signals, although the parameter estimation is poor.

The CBC pipeline was designed to analyse data from a network of detectors to search specifically for gravitational wave signals from binary neutron stars and black holes [9]. It proceeds as follows: first, a bank of templates covering the desired mass range is produced. For the NINJA analysis, we used a template bank covering masses between 20 and $90 M_{\odot}$ with a minimal match of 0.97 . The data from each of the detectors are separately match filtered against the template waveforms [50]. For this analysis, we restricted our attention to the mock data generated for the three LIGO detectors (the $4 \mathrm{~km}$ and $2 \mathrm{~km}$ detectors denoted $\mathrm{H} 1$ and $\mathrm{H} 2$, respectively, at Hanford, WA and the $4 \mathrm{~km} \mathrm{L1} \mathrm{detector} \mathrm{at} \mathrm{Livingston,} \mathrm{LA).} \mathrm{A} \mathrm{trigger} \mathrm{is}$ produced whenever the signal-to-noise ratio exceeds the desired threshold of 5.5. A coincident trigger is recorded whenever there are triggers from two or more detectors with comparable masses and coalescence times [51]. Finally, these coincident triggers are subjected to a set of signal based vetoes, in particular the $\chi^{2}$ [52] and $r^{2}$ [53] tests, designed to separate signals from non-stationary transients in the noise. The parameters used here were chosen, where possible, to match those used in the analysis of the LIGO data performed in [10].

Our results are summarized in table 1 which shows the number of injections recovered by the analysis pipeline at each stage of the analysis for the two searches described here. The EOB search is capable of detecting a greater fraction of the simulated signals than the TaylorF2 templates truncated at LSO. This is further highlighted in figure 6 where we show those simulated signals which were recovered by the two different waveform families. The EOB model clearly performs better, particularly at higher masses. This is consistent with the findings of the previous section. 

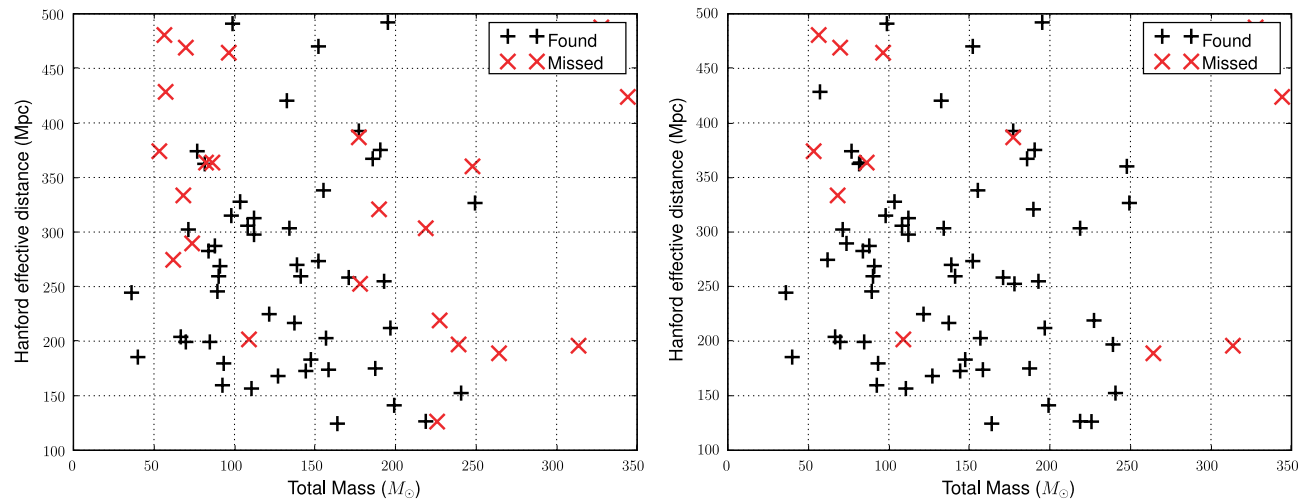

Figure 6. Found and missed numerical injections for the TaylorF2 (left panel) and EOB (right panel) searches of the NINJA data. The found and missed injections are plotted on the total mass, Hanford effective distance plane. The effective distance for a detector provides a measure of the amplitude of the signal at that site, taking into account the distance and orientation of the source. For both searches, the majority of the close simulations are recovered. EOB templates are seen to perform better, particularly at higher masses. In both searches, the simulation with total mass $105 M_{\odot}$ and effective distance of $200 \mathrm{Mpc}$ is missed. This has a peak amplitude at the start of the waveform, rather than coalescence, and although a coincident trigger is recorded the time between it and the waveform peak is too large for our algorithm to associate them.

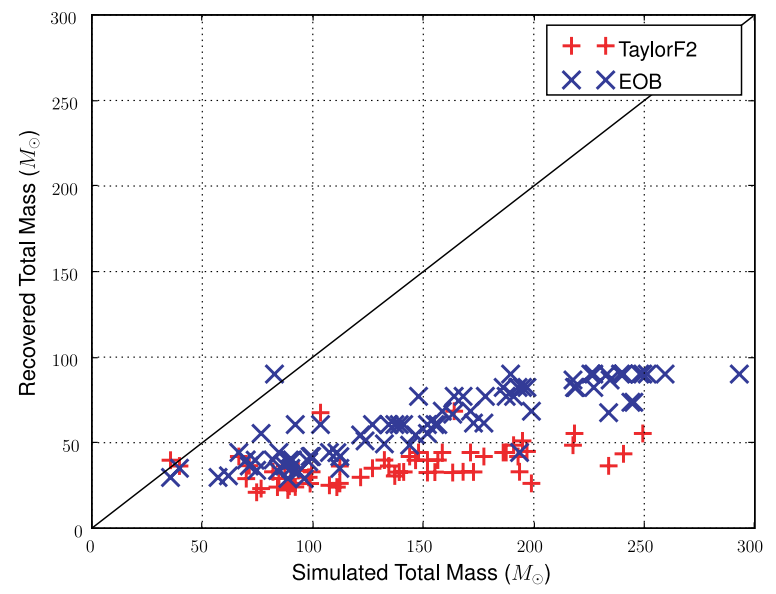

Figure 7. Accuracy of recovering the total mass of simulated signals for the TaylorF2 (red + ) and EOB (blue $\times$ ) models. For both of the searches, the total mass is estimated poorly and systematically lower than the simulated mass. This is due to the fact that the search has been performed with inspiral only waveforms for which the search extends only up to $90 M_{\odot}$.

Next, we turn to parameter estimation. Figure 7 shows the accuracy with which the total mass of the simulated signals is recovered using the inspiral only waveforms. For both the TaylorF2 and EOB models, the parameter recovery is poor, particularly at higher masses. This is to be expected, since we are searching with partial waveforms and, at the higher masses, it is the merger and ringdown of the simulations which occupies the sensitive band of the detectors. Furthermore, the template bank extends only to a total mass of $90 M_{\odot}$ making accurate parameter recovery of the high-mass simulations impossible. 


\section{Discussion}

In the coming years, the first detection of gravitational waves from coalescing binaries will surely be achieved. Following the first detection, attention will focus on extracting as much astrophysical information as possible from the observed signal. In the studies described here, we have addressed the ability to perform both the detection and parameter estimation problems using template waveforms which cover only part of the binary coalescence. We have made use of two different waveforms-the TaylorF2 PN waveforms taken to LSO and the EOB waveforms to light ring. In addition, we have used two different methods to evaluate the detection and parameter estimation capabilities of these signals - a Monte Carlo study using EOBNR waveforms as the 'true' signal, and an analysis of numerical relativity waveforms in the NINJA data. In all cases, the conclusion is the same: the inspiral only templates are useful for detection of the signal, but do not provide good parameter estimation, particularly for the higher mass signals. This is to be expected as, for high-mass binaries, it is the merger and/or ringdown which occurs at the most sensitive frequency of the detectors. We observe that the EOB waveforms perform somewhat better than the TaylorF2 waveforms. This is expected as the EOB waveforms extend to the light ring and therefore capture the plunge part of the waveform which is not incorporated in the TaylorF2 PN model.

The results of this study show that post-Newtonian-based inspiral only waveforms will not be sufficient for satisfactory detection and parameter estimation of higher mass black hole binaries. Full waveforms derived from a synthesis of post-Newtonian waveforms and numerical relativity results, such as the EOBNR model [14-18], or phenomenological models, such as $[46,47]$, will be necessary.

\section{Acknowledgments}

We thank Duncan Brown, Alessandra Buonanno, Mark Hannam, Sascha Husa, Badri Krishnan, Larne Pekowsky and Lucia Santamaria for helpful comments on the analysis and paper. We are grateful to Alessandra Buonanno, Evan Ochsner and Craig Robinson for discussions on and coding up the EOBNR model. We thank the Kavli Institute for Theoretical Physics (KITP) Santa Barbara for hospitality during the workshop 'Interplay between Numerical Relativity and Data Analysis', where the NINJA project was initiated; the Kavli Institute is supported by NSF grant PHY 05-51164. BS acknowledges the support of the UK Science and Technology Facilities Council. SF was funded by the Royal Society. BF was funded by the International REU program and grant NSF-0838740.

\section{References}

[1] Sathyaprakash B and Dhurandhar S 1991 Phys. Rev. D 44 3819-34

[2] Blanchet L 2002 Living Rev. Rel. 53 (arXiv:gr-qc/0202016)

[3] Blanchet L, Faye G, Iyer B R and Sinha S 2008 Class. Quantum Grav. 25165003 (arXiv:0802.1249 [gr-qc])

[4] Blanchet L, Faye G, Iyer B R and Joguet B 2002 Phys. Rev. D 65 061501(R) Blanchet L, Faye G, Iyer B R and Joguet B 2005 Phys. Rev. D 71 129902(E) (erratum) (arXiv:gr-qc/0105099)

[5] Blanchet L, Damour T, Esposito-Farèse G and Iyer B R 2004 Phys. Rev. Lett. 93091101 (arXiv:gr-qc/0406012)

[6] Lsc algorithm library http://www.lsc-group.phys.uwm.edu/lal

[7] Abbott B et al (LIGO Scientific) 2004 Phys. Rev. D 69122001 (arXiv:gr-qc/0308069)

[8] Abbott B et al (LIGO Scientific) 2005 Phys. Rev. D 72082001 (arXiv:gr-qc/0505041)

[9] Abbott B et al (LIGO Scientific) 2008 Phys. Rev. D 77062002 (arXiv:0704.3368)

[10] Abbott B et al (LIGO Scientific) 2008 Search for gravitational waves from low-mass binary coalescences in the first calendar year of s5 ligo data arXiv:0901.0302v3 [gr-qc]

[11] Buonanno A and Damour T 1999 Phys. Rev. D 59084006 (arXiv:gr-qc/9811091) 
[12] Buonanno A and Damour T 2000 Phys. Rev. D 62064015 (arXiv:gr-qc/0001013)

[13] Buonanno A, Cook G B and Pretorius F 2007 Phys. Rev. D 75124018 (arXiv:gr-qc/0610122)

[14] Damour T and Nagar A 2008 Phys. Rev. D 77024043 (arXiv:0711.2628)

[15] Damour T, Nagar A, Dorband E N, Pollney D and Rezzolla L 2008 Phys. Rev. D 77084017 (arXiv:0712.3003)

[16] Damour T, Nagar A, Hannam M, Husa S and Bruegmann B 2008 Phys. Rev. D 78044039 (arXiv:0803.3162)

[17] Buonanno A et al 2007 Phys. Rev. D 76104049 (arXiv:0706.3732)

[18] Boyle M et al 2008 High-accuracy numerical simulation of black-hole binaries: computation of the gravitational-wave energy flux and comparisons with post-Newtonian approximants Phys. Rev. D 78104020 (arXiv:0804.4184)

[19] Aylott B et al 2009 Class. Quantum Grav. arXiv:0901.4399 submitted

[20] Hannam M, Husa S, Sperhake U, Bruegmann B and Gonzalez J A 2008 Phys. Rev. D 77044020 (arXiv: 0706.1305)

[21] Hannam M, Husa S, Bruegmann B and Gopakumar A 2008 Phys. Rev. D 78104007 (arXiv:0712.3787)

[22] Tichy W and Marronetti P 2008 Phys. Rev. D 78081501 (arXiv:0807.2985)

[23] Marronetti P, Tichy W, Bruegmann B, Gonzalez J and Sperhake U 2008 Phys. Rev. D 77064010 (arXiv: 0709.2160)

[24] Tichy W and Marronetti P 2007 Phys. Rev. D 76061502 (arXiv:gr-qc/0703075)

[25] Pollney D et al 2007 Phys. Rev. D 76124002 (arXiv:0707.2559)

[26] Rezzolla L et al 2008 Astrophys. J. 679 1422-6 (arXiv:0708.3999)

[27] Vaishnav B, Hinder I, Herrmann F and Shoemaker D 2007 Phys. Rev. D 76084020 (arXiv:0705.3829)

[28] Hinder I, Vaishnav B, Herrmann F, Shoemaker D and Laguna P 2008 Phys. Rev. D 77081502 (arXiv:0710.5167)

[29] Pretorius F and Khurana D 2007 Class. Quantum Grav. 24 S83-S108 (arXiv:gr-qc/0702084)

[30] Boyle M et al 2007 Phys. Rev. D 76124038 (arXiv:0710.0158)

[31] Scheel M A et al 2009 High-accuracy waveforms for binary black hole inspiral, merger, and ringdown Phys. Rev. D 79024003 (arXiv:0810.1767)

[32] Etienne Z B, Faber J A, Liu Y T, Shapiro S L and Baumgarte T W 2007 Phys. Rev. D 76101503 (arXiv: 0707.2083)

[33] Bruegmann B et al 2008 Phys. Rev. D 77024027 (arXiv:gr-qc/0610128)

[34] Husa S, Gonzalez J A, Hannam M, Bruegmann B and Sperhake U 2008 Class. Quantum Grav. 25105006 (arXiv:0706.0740)

[35] Koppitz M et al 2007 Phys. Rev. Lett. 99041102 (arXiv:gr-qc/0701163)

[36] Imbiriba B et al 2004 Phys. Rev. D 70124025 (arXiv:gr-qc/0403048)

[37] van Meter J R, Baker J G, Koppitz M and Choi D I 2006 Phys. Rev. D 73124011 (arXiv:gr-qc/0605030)

[38] Zlochower Y, Baker J G, Campanelli M and Lousto C O 2005 Phys. Rev. D 72024021 (arXiv:gr-qc/0505055)

[39] Campanelli M, Lousto C O, Marronetti P and Zlochower Y 2006 Phys. Rev. Lett. 96111101 (arXiv: gr-qc/0511048)

[40] Sperhake U 2007 Phys. Rev. D 76104015 (arXiv:gr-qc/0606079)

[41] Pretorius F 2005 Class. Quantum Grav. 22 425-52 (arXiv:gr-qc/0407110)

[42] Pretorius F 2005 Phys. Rev. Lett. 95121101 (arXiv:gr-qc/0507014)

[43] Scheel M A et al 2006 Phys. Rev. D 74104006 (arXiv:gr-qc/0607056)

[44] Damour T, Iyer B R and Sathyaprakash B S 1998 Phys. Rev. D 57 885-907

[45] Baker J G, Centrella J, Choi D I, Koppitz M and van Meter J 2006 Phys. Rev. Lett. 96111102 (arXiv: gr-qc/0511103)

[46] Ajith P et al 2008 Phys. Rev. D 77104017 (arXiv:0710.2335)

[47] Ajith P et al 2007 Class. Quantum Grav. 24 S689-700 (arXiv:0704.3764)

[48] Babak S, Balasubramanian R, Churches D, Cokelaer T and Sathyaprakash B S 2006 Class. Quantum Grav. 23 5477-504 (arXiv:gr-qc/0604037)

[49] Cokelaer T 2007 Phys. Rev. D 76102004 (arXiv:0706.4437)

[50] Allen B, Anderson W G, Brady P R, Brown D A and Creighton J D E 2005 FINDCHIRP: an algorithm for detection of gravitational waves from inspiraling compact binaries arXiv:gr-qc/0509116

[51] Robinson C A K, Sathyaprakash B S and Sengupta A S 2008 A geometric algorithm for efficient coincident detection of gravitational waves Phys. Rev. D 78062002 (arXiv:0804.4816)

[52] Allen B 2005 Phys. Rev. D 71062001 (arXiv:gr-qc/0405045)

[53] Rodríguez A 2007 Reducing false alarms in searches for gravitational waves from coalescing binary systems Master's Thesis Louisiana State University http://www.ligo.caltech.edu/docs/P/P070056-00.pdf 